\title{
La parola di piombo: Il peso della stampa nell'avventura culturale di Tomaso Garzoni
}

\author{
Francesco Guardiani
}

L'occasione di questo intervento è la pubblicazione, non più recentissima, delle Opere di Tomaso Garzoni, a cura di Paolo Cherchi (1993). La misura del volume, oltre settecento pagine, impone tempi lunghi sia per l'assimilazione di una gran messe di dati testuali che per la necessaria riflessione su di essi. Non ci si può accostare a un autore del genere, e per di più con un'opera che comprende tre quarti del suo corpus, senza sentire l'esigenza di dare un senso alla sua avventura culturale tutt'intera, che si colloca in un momento cruciale della nostra tradizione letteraria, in un affollatissimo secondo Cinquecento ricco di umori e di tendenze nuove ed estreme, con opere di vasta erudizione e larga diffusione.

Con buon ritardo, e col proposito di un chiarimento di ordine teorico, raccolgo allora la proposta implicita del Cherchi, organizzando il discorso in questo modo: do una descrizione, svelta per quanto possibile, del volume e quindi delle opere singole che vi sono contenute, per poi soffermarmi brevemente a considerare l'ideologia dell'autore; da qui risalgo all'ampio contesto culturale in cui tale ideologia ha da collocarsi e propongo, infine, per questa cultura in generale e per l'esperienza di Garzoni in particolare, l'impiego della teoria delle trasformazioni culturali di Marshall McLuhan che più e meglio di ogni altro, a mio avviso, ha studiato la natura del mezzo stampa e le sue profonde implicazioni. Ovviamente un impegno del genere, soprattutto per quanto concerne l'ultimo punto, comporta una buona dose di fiducia nella disponibilità di chi legge ad accettare come assioma di base il determinante rilievo della stampa nell'atto della scrittura. Non di questo mi occupo quindi, ma piuttosto del modo in cui tale rilievo viene a materializzarsi sulla pagina del Garzoni e dei suoi contemporanei.

\section{Le opere}

Il volume di Cherchi è in effetti una seconda edizione, ma "rifatta dalle fondamenta" (29), approntata nell' ambito delle celebrazioni per il quarto centenario della morte del Garzoni (1549-1589). La prima, sempre per le cure dello stesso studioso, fu pubblicata nel 1972 dalla benemerita, ma sfortunata, casa editrice napoletana Fulvio Rossi e, per la cessazione dell'attività della stessa, ebbe scarsissima diffusione. Si ripresentano dunque le stesse opere dello scrittore di Ba- 
gnocavallo: quattro intere, Il teatro dei vari e diversi cervelli mondani (1583), L'ospidale dei pazzi incurabili (1586), La sinagoga degli ignoranti (1589), e Il mirabile cornucopia consolatorio (1601), e una ridotta, La piazza universale di tutte le professioni del mondo (1585). Della Piazza, ritenuta l'opera maggiore, sempre il Cherchi, con la collaborazione di Beatrice Collina, ha completato l'edizione critica che è ora in corso di stampa per la collana dei "Millenni" Einaudi. Mancano nel novero Le vite delle donne illustri della Scrittura Sacra (1586), di cui abbiamo una recente edizione a cura di Beatrice Collina, L'huomo astratto (1604), opera d'ispirazione mistica leggibile oggi in anastatica, e infine Il serraglio de gli stupori del mondo (1613), che è l'opera ultima del Garzoni (inizialmente intitolata $I l$ palazzo de gli stupori . . . , un fatto non trascurabile nell'architettura compositiva del Nostro) ampiamente rimaneggiata e portata a compimento da Bartolomeo Garzoni, fratello dell'autore e, come lui, canonico lateranense; del Serraglio abbiamo solo, a tutt'oggi, la stampa secentesca.

Un'occhiata alle date di pubblicazione e a quelle della vita del Garzoni, morto nell' 89 a soli quarant'anni, dà subito un'idea della rapidità straordinaria con cui egli riusciva a realizzare i suoi ambiziosissimi progetti (fra i quali sono da includere anche quelli di carattere filologico, come la traduzione del Colloquio di Dionigi il Certosino, 1583, e l'edizione della Hugonis de Sancto Victore Opera tribus tomis digesta, 1588). È un elemento su cui si dovrà tornare; basti per ora dire che per quanto prodigioso potesse essere "il cervello dell'auttore", descritto dallo stesso nell'introduzione alla prima ristampa del Teatro (1585), la rapidità di composizione non si spiega senza tener conto delle caratteristiche tipografiche di una tale scrittura, che non è quindi solo un fatto individuale, ma appartiene a un ambiente e a una cultura.

Il Teatro ha una struttura biologica, o botarica, per così dire, che richiama l'albero medievale delle scienze: "Io ritrovo che, a guisa ch'arbore o pianta in vari tronchi principali di divide, e que' tronchi partiscono in vari e diversi rami, così è partito questo nome di cervello in vari significati, anzi specie di cervelli nominati al mondo" (49). Dal tronco principale si dipartono i vari rami. Abbiamo i cervelli generici prima, divisi in "quieti e riposati", "bravi ed armigeri", "gioviali ed allegri", "faceti", "arguti", "accorti astuti e trincati", "vivaci pronti e svegghiati", "sottili acuti e giudiziosi", "saputi ed intelligenti", virtuosi e nobili". Ci sono poi i "cervellini" distinti anch'essi in sottocategorie, cui fanno seguito "scemando ancor più" quelli che "si dimandano cervelluzzi"(50) e, quindi, in serie sempre nutritissime, i "cervelletti", i "cervelloni" e i "cervellazzi". L'ordine generale di presentazione, dai piccoli ai grandi, dai grandi ai cattivi, non è di per sé rilevante. Conta invece l'atteggiamento parcellizzante e riduttivo dell'autore che, all'interno di ognuno dei primi contenitori, ripartisce la materia in sottocategorie le quali, a loro volta, sono descritte da intere serie di proprietà o attributi; e questi, per finire, sono illustrati da catene di aneddoti e citazioni. Valga un esempio fra mille: 
Dimostrasi un cervello faceto communemente in cinque cose: nelle sentenze o detti, ne' proverbi, ne' motti, nelle risposte e ne' concetti. Nelle sentenze: come talor ci dimostrò Diogene, chiamando i ricchi ignoranti "pecore della lana d'oro" e la gioventù bella ma viziosa "un sontuoso albergo abitato da un brutto forestiere". $\mathrm{Ne}$ ' proverbi: come quel faceto cervello che disse proverbiosamente al suo signore (il quale mormorava de' vizi de' moderni sudditi) che 'l pesce comincia a putir dal capo; e che di più, che tale è la cagnola quale è la signora. Ne' motti: come quel Filosseno il quale, essendo in una cena dove era da' servitori portato in tavola pane negro, disse, facetamente motteggiando il signore: "Di grazia signore non me ne fate portar molto, acciò le tenebre non avanzino i lumi". Nelle risposte: come quel Pontidio romano, al quale essendo dimandato: "Che uomo ti pare uno che sia trovato in adulterio?", rispose: "Lento". Ne' discorsi o concetti: come quello del Bembo il quale, appresso il Castiglione, discorse intorno alla sciocchezza di quel podestà fiorentino che fece intendere a' suoi nemici che, se perseveravano a far la batteria sì aspra alla Castellina, egli ancora l'avrebbe fatta alla disperata, ponendo il tosco sopra le balle dell'artigliaria, e sparandole a quella maniera. Concetto faceto fu quello di Luigi Groto ancora, quando, chiesto dalla sua donna di dover basciare una fanciullina sua, spiegolle il seguente madrigale:
Madonna, se volete
ch'un dono in nome vostro io porti altrui, convien ch'io prenda il don prima da vui.
Però, s'or mi chiedete,
ch'a la fanciulla vostra un bacio i' dia,
da voi convien, ch'io lo riceva pria. (68)

Colpisce l'entusiasmo con cui l'autore anima le sue sterminate filatesse. Garzoni ha il tono energico dello scopritore, o meglio del rivelatore delle meraviglie del mondo. Che sono poi meraviglie cartacee, riposte tutte negli scaffali di una ipotetica biblioteca dell'intera umanità cui alla sua sola forza demiurgica è dato il privilegio di accedere e attingere. Ecco allora, logica mente, il vanto del proprio cervello. Nella fucina di Vulcano, Giove è impegnato col suo seguito al conio dei vari e diversi cervelli del mondo. Arriva il turno di Garzoni e

L'auttore presente si ricorda di questo: che arditamente si fece innanzi e chiese per grazia dai dei che gli fosse dato cervello in tal porzione che, usandolo in cose gravi ed anco in cose facete, potesse dar delle loro grazie al mondo quella contezza che di loro e di lui paresse degna. Ove i benigni dei gli fecer largo dono d'un cervello disposto a qualunque sorta di curiosità e di gravità insieme che da lui tentata fosse, aggiongendo per special lor grazia a tal cervello molte altre qualità che dai scritti e dalla conversazione sua possono esser note e manifeste a moltissime persone. Mercurio si compiacque di farlo studioso della eloquenza, Apollo dell'una e dell'altra poesia, Minerva della sapienza scritturale, Minòs della legge. Ma Proteo se gli dimostrò più partigian d'ogn'altro, disponendolo a trasformar se stesso in qualunque professione che al suo appetito piacesse, talché potrebbesi forsi annoverar l'auttore 
per cervello universale, avendo già mostrato in scritto che poche son le cose delle quali non possa egli così mediocremente almen discorrere e ragionare. (54-55)

Di fronte a questa autoesaltazione e alla valanga di dati eruditi che con sprezzatura principesca l'autore regala in stile tutt'affatto informale ai suoi numerosi e grati lettori, non si può non rimanere ammirati e chiedere come tanta cultura originasse da una sola mente. Il formidabile lavoro filologico del moderno curatore chiarisce l'arcano. La "libridine" del canonico, così chiama Cherchi il fervore compositivo del Garzoni, è in realtà fondata su poche fonti intermedie, tutte invariabilmente enciclopediche come, per il Teatro, la Polyanthea di Domenico Nanni Mirabelli e soprattutto l'Officina di Ravisio Testore. C'è una differenza notevole, però, tra le opere a monte e quelle a valle. Ravisio Testore (14801524), per esempio, è un tardo umanista francese, un compilatore di materiale classico in cui è facile scorgere una motivazione ancora legata agli alti ideali del passato. Garzoni invece punta tutto sull'oggi, sulle esigenze di informazione di un nuovo, già vastissimo e crescente, pubblico alfabetizzato dalla stampa e non del tutto o non affatto a suo agio con i paludamenti classici e la lingua latina.

Al Teatro dei vari e diversi cervelli mondani segue L'ospidale dei pazzi incurabili, un altro spazio architettonico che si presta bene a un sistematico frazionamento atomistico: il luogo è diviso in celle in ognuna delle quali si trovano esempi di un tipo di pazzia. Si tratta, per cominciare, di pazzi veri, ché Garzoni "è avverso a quella tradizione erasmiana che esalta la pazzia paolina e la dotta ignoranza"(247); e forse questo spiega l'interesse straordinario per il volume, testimoniato da numerose traduzioni e ristampe, da parte di medici e scienziati. Anche qui Garzoni fa essenzialmente opera di mediazione. Oltre a riattingere alla solita Officina del Testore, ci informa Cherchi, egli sfrutta appieno le risorse dell' "aneddotica paesana che era stata invece tenuta schifiltosamente lontana dai registri colti del Teatro" (248). Non abbiamo solo, allora, un Garzoni traduttore e riordinatore di vecchie enciclopedie, ma un operatore culturale voracissimo, attento a tutti gli aspetti della vita del suo tempo, che attinge a ogni sorta di fonte elencativa. I ritratti che si colgono qui, per ogni deformazione mentale, sono anche da collegare a un gusto tipico dell'età per il diverso, il grottesco, l'assurdo e il mostruoso, per il rovescio del normale insomma, di cui si possono vedere molti esempi, mi pare utile ricordarlo, nel Mondo alla rovescia di Giuseppe Cocchiara. Valga a esempio di tutte le pazzie quella d'amore, in cui ritroviamo il nostro autore con le sue partizioni della materia e le sue filatesse.

Questa pazzia si mostra d'essere radicata principalmente ne' pensieri, ne' desideri, ne' concetti, nelle risoluzioni, nelle parole, ne' gesti, ne' cenni e nelle azioni . . Co' folli pensieri tende l'insano amante a fare castelli in aria da se stesso . . . e così, dilatandosi in mille pensieri di trovar ruffiani, comari, servitori, pizzochere, balie, massare; di scriver lettere, polize, sonetti, madrigali, canzoni; di mandar fiori, mazzetti, presenti, mance, donativi . . . Co desideri stolti brama talora d'essere uno pulice o una mosca o veramente una formica per entrare nella camera della sua 
amante . . . Co' concetti va formando imprese amorose, motti leggiadri e vaghi, rime dolci e soavi, sentenziosi parlari, artificiosi detti, stratagemmi politi; e fabrica nell'animo, giorno e notte, quanto pensa dovergli apportare giovamento nel suo fine. Con le risoluzioni determina di vederne il fine, e dar giovamento a' suoi pensieri, risolvendosi di non stare più, di non voler patire più affanni, di non soffrire più tormenti, ma vedere ciò che dice, ciò che pensa, ciò che si risolve. Con le parole l'affronta e le ragiona or garbo or dolce or di meggio sapore. Co' gesti la move a compassione ponendo le braccia in croce, e la fa struggere di pietà quando sa fare co' cenni e con l'azioni. Finalmente si diporta in modo che le bestie sono qualche volta più sagge e più prudenti che non è uno di questi pazzi d'amore. (314-15)

Della Sinagoga (termine che ha sì significato di "congregazione", come ci dice il curatore, ma che reca anche in sé un marcatissimo connotato dispregiativo per l'associazione agli ebrei e, in generale, agli eretici, con tutto ciò che poteva comportare in pieno furore controriformistico) non si dirà molto. Le fonti sono più erudite: gli Adagia di Erasmo e gli Hieroglifica del Pierio, ovvero Giovanni Pierio Valeriano Bolzani (377). La motivazione a scrivere un'opera del genere è tutta particolare e va senz'altro registrata.

La Sinagoga è un'opera di violenta ritorsione contro un 'gallo', cioè un francese che rimane innominato. Ma questo 'gallo' è niente meno che il grande filologo Giusto Giuseppe Scaligero il quale . . scrisse una . . . recensione severissima degli Scholia al primo libro del De oratore ciceroniano di Fabio Paulini, l'umanista udinese (m. nel 1605) amico del Garzoni (375).

Lo Scaligero non era affatto nuovo a queste imprese; ma al di là delle sue ragioni e del suo temperamento, qui si deve notare che non era il solo in quei tempi ad attaccar brighe ché, anzi, la disputa è un evento tipico della cultura cinquecentesca. Ricordiamo quelle tra Aretino e Franco, Aretino e Doni, Scaligero ed Erasmo, Scaligero e Cardano, Tasso e la Crusca, e infine la doppia appendice secentesca: le contese del Marino col Murtola e con lo Stigliani. La Sinagoga, ci dice il Cherchi, presenta una compattezza e un rigore artistico che le altre opere non hanno (378). Eppure fu, tra le opere garzoniane, "la meno stampata e apprezzata, forse per la natura erudita dei suoi materiali e per la mancanza di nitide classificazioni di aneddoti" (379). La seconda ragione mi pare più probante: è la segmentazione concisa, netta e cumulativa che il pubblico del secondo Cinquecento gradisce di più, come si dirà meglio sotto.

Del brevissimo Mirabile cornucopia consolatorio si ricorderà, innanzi tutto, che appartiene al genere degli encomi paradossali. Si tratta di una serie di lodi delle corna scritta in forma di lettera indirizzata a un amante vecchio fatto becco. Mi limito a notare, per riconoscere la matrice onniavolgente della stampa, l'assenza di problematicità ideologica per la compresenza nel corpus garzoniano di questa opera burlesca e di altre che trattano materia più alta e idealizzata, fino alla scrittura mistica dell'Huomo astratto. 
Resta da dire della manciata di capitoli della Piazza universale di tutte le professioni del mondo, l'opera maggiore, più nota e fortunata di Garzoni; che è poi anche la più estesa, scritta, secondo la testimonianza dell'autore, in soli sei mesi. Pare impossibile,

eppure non si tratta di un vanto: chi sa come il Canonico lavorava può credergli senz'altro perché sa che dove più abbonda l'erudizione tanto più opera il rampino del plagiario a rubare testi altrui. La Piazza non contiene ricerche di prima mano, ma è un mosaico di testi altrui, un centone di dati presi di peso da lavori altrui, un intarsio di citazioni ricavate in percentuale altissima da testi intermediari: in tal senso la Piazza acquista un valore paradigmatico rappresentando il caso più oltranzoso, per l'estensione e per la qualità, di quel fenomeno di riscrittura che fu tanto diffuso nel secondo Cinquecento. (544)

L'appuntamento di lettura che ci è dato dall'annuncio della imminente pubblicazione dell'edizione critica della Piazza ci esime per ora da un ragguaglio sui capitoli di questa scelta. Va però notata la fondamentale dimensione ideologica di quest'opera.

\section{L'ideologia del Garzoni e il contesto "tipografico"}

La Piazza è una risposta allo scetticismo rinascimentale che ebbe nel De incertitudine et vanitate scientiarum di Agrippa di Nettesheim un testo privilegiato di riferimento. La controproposta del Garzoni con la Piazza è ottimistica, fattiva, indubbiamente moderna e, oserei addirittura, fondativa di una nuova etica del lavoro individuale nel più ampio contesto sociale. Tale almeno dovette apparire in Germania dove fu presto tradotta e ripetutamente ristampata nel corso del Seicento. In Garzoni le professioni del mondo sono le forze che lo rendono un teatro di creatività e di progresso. La ricchezza e la varietà dell'elenco dei mestieri si moltiplica quando si parla degli strumenti, dei materiali e dei modi di lavorazione: una vera esplosione di energia produttiva. Ideologicamente la Piazza non serve solo a legittimizzare come utili e necessarie tutte le attività umane, ma anche e soprattutto a certificare che la nuova cultura è fondata sulla specializzazione. Non è un sognante filosofo platonico, un teorizzatore astratto, l'uomo nuovo alle soglie dell'universo scientifico del secolo che sarà di Galileo e di Cartesio, e neanche un generico homo faber, ma un produttore di oggetti particolari, integrato in un reticolo di relazioni di ambiente e di categorie di lavoro. Ecco, tra i centocinquantacinque mestieri che affollano la Piazza, un elenco parziale che risulta da una sezione della lettera " $c$ " nell'indice: "cernitori di lana, cestari, cestaruoli, chiavari, chiodaruoli da panni di lana, chiromanti, cialdonai, ciavatini, cifranti, cimatori da lana, cerugici, ciurmatori, cocchieri, comari, comici, comandatori, commentatori, compositori di libri, computisti, professori di concili, confertinari, consiglieri, contadini" (545).

Già si è accennato sopra, a proposito dell'Ospidale, alla reazione di Garzoni alla corrente scettica erasmiana, e s'è detto anche della sua lettura interessata dei 
famosi Adagia per la Sinagoga. Tutto questo, e l'atteggiamento tenuto nella febbrile composizione della Piazza, indicano con chiarezza che l'autore è tutt'altro che una figura di sfondo nel grande dibattito filosofico cinquecentesco sull'utilità della scienza e sull'eredità dell'umanesimo. Egli è invece un "critico militante", cosciente e aggiornato, che reagisce con i fatti, ovvero con opere destinate alla più ampia diffusione, a una posizione ideologica fondamentalmente scettica e rafforzata da un nuovo stoicismo di marca senechiana. In pagine memorabili e attualissime di Enciclopedismo e riscrittura, Cherchi dimostra che il preciso obiettivo polemico del Garzoni è il De incertitudine di Agrippa di Nettesheim (58-67); l'opera era stata tradotta da Ludovico Domenichi nel 1547 e, più volte ristampata, aveva suscitato interesse e solidarietà in Italia in Anton Francesco Doni e in altri "scapigliati" (61).

Per allargare l'orizzonte culturale che stiamo descrivendo, fermiamoci un attimo a considerare il caso del Doni (1513-1574), cui tra l'altro ci invita la recente ricerca di Giuseppe Candela, Manierismo e condizioni della scrittura in Anton Francesco Doni. È fin troppo facile accostarlo a Garzoni, non solo per la prossimità cronologica, ma soprattutto per il suo operare a vasto raggio, per quanto riguarda temi e propositi divulgativi, con il nuovo mezzo della stampa. Doni usa inoltre la stessa tecnica, se così possiamo chiamarla, della parcellizzazione della materia trattata. In "Poligrafismo e ideologia", il capitolo con cui il Candela conclude il suo lavoro, il poligrafismo doniano non è tanto una scelta quanto una realtà moderna e irreversibile che giustifica l'attacco ai pedanti, le simpatie erasmiane e il radicalismo "socialista". Ma la massiccia trasformazione culturale di cui Doni si fa interprete e promotore è tale che alla fine conduce a uno iato incolmabile fra le aspirazioni rivoluzionarie dello scrittore e la realtà caotica che gli sta innanzi. E da qui che nasce la sua rinuncia all'azione, il suo ritiro dalla scena, il suo pessimismo scettico (cfr. Candela, "Gli anni del ritiro" 137-150). Il Doni muore nel ' 74 quando Garzoni ha venticinque anni; Il teatro dei vari e diversi cervelli mondani, la sua prima opera, è di nove anni dopo. Si può, credo, imputare anche alla distanza generazionale la maggiore sistematicità del Garzoni, che è del tutto svincolata da propositi eversivi e, anzi, si propone come restaurativa dei valori dell'umanesimo. Ma il "superamento" di ordine ideologico del Garzoni non deve trarre in inganno se si guarda al fronte culturale più ampio: la percezione della realtà in maniera frazionata e cumulativa, imposta a entrambi gli scrittori dalla coscienza di operare con il mezzo nuovo della stampa, li rende solidali nel proiettarsi verso la modernità.

Una volta riconosciuta l'avvolgente presenza della stampa come elemento determinante dell'ambiente culturale del tempo, lo shift ideologico che abbiamo notato tra il Doni e il Garzoni ci invita a distinguere diversi momenti di applicazione della stampa e quindi a notare la diversità dei suoi effetti registrabile sull'asse cronologico. L'esordio del Doni sulla scena letteraria con un volume di Lettere nel 1544 è rivelativo in questo senso. Solo sette anni prima erano uscite le famose Lettere dell' Aretino aprendo la nutritissima serie del "genere", come ben 
certifica il Quondam: "Tra il 1538 e il 1627 vengono pubblicati 540 volumi che raccolgono lettere di autori contemporanei . . . . L'atto fondativo di questo genere tutto editoriale . . è legato al nome di Pietro Aretino" (676). Le Lettere del Doni non sono di qualità inferiore a quelle del "maestro", ci dice il Candela (113), né, supponiamo, lo saranno state molte di quelle successive. Eppure non c'è paragone tra le prime e le altre, se si guarda alla risposta dei lettori. Ho tentato di spiegare la sprorporzione a tutto vantaggio dell'Aretino in un lavoro recente ora in corso di stampa presso la casa editrice Salerno, "Aretino e Rabelais figure aurorali della modernità" (Atti del convegno internazionale su Pietro Aretino. Roma-Toronto-Los Angeles, 1992). Ad esso rimando, ricordando qui che la carta delle lettere è giocata dall'Aretino con puro istinto e che la vincita è enorme perché il pubblico, non ancora avvezzo allo scandaglio sistematico della materia trattata, come lo sarà in Garzoni, pensa ancora alla figura mitica dello scrittore dell'età del manoscritto; scrittore che però nelle Lettere è vicinissimo, reale, vivente. L'epifania è nella forma e lo shock è inevitabile; ma la forma è presto normalizzata nella ricezione del pubblico e le Lettere del Doni non possono suscitare lo stesso scalpore. Nel corso del Cinquecento è in rapidissima trasformazione non solo l'effetto della stampa sulla scrittura, ma anche l'effetto della stampa sui suoi fruitori, sul crescente numero di lettori alfabetizzati che non hanno ancora l'icona publica dell" "uomo di lettere", una realtà emergente che sarà definita soltanto molto più tardi. Ecco, a proposito, McLuhan: "As for the role of the author [nell'età della prima stampa], it did not exist, so the writer tried on various masks of preacher and clown for the first two centuries, discovering the role of "man of letters' only in the eighteenth century" (195).

\section{Il mezzo stampa e i suoi effetti}

Nelle pagine precedenti ho individuato e isolato gli elementi che occorrono per sostenere la proposta di un ricorso a McLuhan. Eccoli: l'operare nell'ambito di una cultura bilingue, latina e italiana; la rapidità del comporre; la percezione parcellizzata della realtà; l'autoesaltazione del personaggio autore e il suo impegno demiurgico, rivelatore; la ricerca di uno spazio proprio da parte dell'autore che perciò entra facilmente in disputa con gli altri "concorrenti"; la diversità degli effetti della stampa sull'asse cronologico. Sono problemi che a loro volta ne aprono degli altri (periodizzazioni, generi letterari, preferenze tematiche e stilistiche, ecc.). La riduzione del tutto al "peso" della stampa non è un tentativo di soluzione ché questioni particolari vogliono soluzioni particolari, ma una indicazione di prospettiva, senza la quale a me pare comunque che tutti i problemi indicati siano destinati a rimanere tali.

Ci si può intendere subito su alcuni effetti "trasparenti" del mezzo stampa, come sono l'ingrossarsi rapidissimo del pubblico alfabetizzato e la conseguente massificazione e democratizzazione della cultura. Maggiori difficoltà si incontrano quando si cominciano a considerare la natura intrinseca del medium e i condi- 
zionamenti percettivi che esso impone ai suoi fruitori, a chi scrive e a chi legge. Si potrebbe dire subito, per esempio, infilando una decina di riferimenti a messaggi oracolari di McLuhan, che il manoscritto è un mezzo tattile e il libro a stampa è un mezzo visivo, e da qui iniziare poi a trarre le conclusioni. Le quali, comunque, si porterebbero. dietro tutti i dubbi legati all'incertezza e all'approssimazione dell'assioma di base. Partiamo allora da un dato reale, la già segnalata differenza nell'uso della stampa in momenti storici diversi. Il medium rimane lo stesso, ma si ha di esso un diverso uso e una diversa percezione tra l'inizio del Cinquecento e quello del Seicento.

Per arrivare a una matura coscienza del mezzo, Amedeo Quondam parte da Francis Bacon, che nel Novum Organum (1620) associa alla stampa la bussola e la polvere da sparo, invenzioni che hanno cambiato "la faccia e la condizione del mondo" (555). È senz'altro giusto fissare un termine ante quem da cui a ritroso si possano cogliere le parziali anticipazioni, ma proprio a dimostrare quanto sia aperto e democratico il panorama delle scritture e riscritture cinquecentesche sta il fatto che la triplice associazione di Bacon è già formulata da Tommaso Campanella nella zona conclusiva (76) della Città del Sole (1602) e, prima ancora di lui, da Gerolamo Cardano (1501-1576) nella sua autobiografia. Eे un passo che val la pena ricordare; lo leggo nella traduzione di Alfonso Ingegno:

Che cosa c'è di più straordinario dell'uso della polvere da sparo, questa folgore dei mortali, molto più pericolosa di quella dei celesti? E non voglio tacere del grande magnete, che ci guida nella notte tenebrosa per mari senza fine in mezzo a tempeste raccapriccianti. Si aggiunga . . . la scoperta della stampa: costruita dalle mani degli uomini, escogitata dal loro ingegno, è tuttavia in grado di rivaleggiare con $\mathrm{i}$ miracoli degli dei. Non ci resta altro ormai che conquistare il cielo. Ma sarebbe follia dell'uomo dimenticare la vanità del tutto e la nostra ignoranza dei primi principi, seppure sarebbe superbia non ammirare queste scoperte. (141-42)

Una vera esaltazione dei nuovi mezzi che si può intendere anche come una prospettiva allettante e inquietante insieme sui tempi futuri. Nel presente, invece, gli effetti più profondi e psicologici della stampa erano percepiti solo a livello subliminale. Cardano non pensa certo alla stampa come strumento delle sue scoperte quando pubblica la soluzione delle equazioni di terzo grado, subito superata da quella delle equazioni di quarto del suo allievo Ludovico Ferrari; ma il nuovo interesse per l'algebra nel Cinquecento coincide con il primo sistematico impiego della stampa. Sarà una pura coincidenza? Non è forse la percezione analitica favorita, se non imposta, dal nuovo medium? Senza considerare poi che proprio la corsa frenetica alla pubblicazione, al libro a stampa, che vede Cardano in concorrenza con Niccolò Tartaglia, è un fatto tutto tipografico che cancella e supera acquisizioni matematiche che resistevano da millenni (Waters 69-99).

Per quanto dunque venisse intesa l'importanza del nuovo medium, le sue implicazioni non erano affatto chiare e le testimonianze del tempo, di cui Quondam fornisce un nutrito dossier, oltre a ribadire questa realtà non servono a molto se 
non si fa luce sulla mutazione onnicomprensiva che la stampa, come fenomeno epocale, determina nella percezione del reale. McLuhan parla di una vera e propria trasformazione antropologica. Ricordo che il sottotitolo del suo The Gutenberg Galaxy è The Making of Typographic Man. Siamo giunti alla radice del problema, e non è una radice letteraria, ma scientifica e psicologica. Il nostro apparato sensorio non è equamente distribuito in cinque canali comunicativi, ma in due. Da una parte sta la vista, dall'altra stanno gli altri quattro sensi. Per il chiarimento di questo punto fondamentale rimando, oltre che a Laws of Media: The New Science, l'ultimo lavoro di Marshall McLuhan completato dal figlio Eric, agli studi di Derrick de Kerckhove; la bibliografia, qualora si vogliano includere anche le ricerche mediche sulle diverse capacità cognitive dei due emisferi cerebrali, è sterminata e il lettore di buona volontà farà da sé.

Il manoscritto, ripeto, è un mezzo audio-tattile e quindi gestaltico e onnicomprensivo, mentre la stampa è un mezzo visivo, parcellizzante e cumulativo. La visualizzazione, in questi termini, non nasce certo con la stampa, bensì con la scoperta dell'alfabeto. La stampa non è che l'applicazione più sistematica e meccanica dell'alfabeto stesso; ma essa segna un passaggio notevolissimo, in cui cadono i residui valori orali e tattili del medium originario e si insediano in maniera sempre più consapevole quelli visivi. Superato questo scoglio, o meglio accettati questi principi, possiamo finalmente dare un senso forte alla frammentazione della scrittura che abbiamo considerato. Per McLuhan, che va fino in fondo, non ci sono dubbi: il monadismo concettuale imposto dalla stampa è la base stessa della democrazia moderna.

C'è una ragione di ordine pratico per "la crescita della cultura" nel Cinquecento. Il mezzo stampa, come le gigantesche banche dati dei nostri tempi ricondizionati percettivamente dall'elettronica, si impone per la sua capacità di contenere e riciclare intere biblioteche in tempi brevi e in un numero illimitato di copie. $\mathrm{Ma}$ il medium, considerato soltanto in questi termini di disponibilità materiale, non spiega affatto la voracità cognitiva dei suoi primi fruitori, non spiega affatto l'entusiasmo con cui venivano recepite le enciclopedie del Garzoni. Non c'era solo un bisogno puro e semplice di conoscenza, ma un bisogno di essere nel presente reinterpretando il passato come in tutte le epoche di grandi trasformazioni culturali, inclusa la nostra. E il passato non poteva che presentarsi in maniera "visually biased", ovvero sistematicamente e soggettivamente "ridotto a pezzi". Questa "riduzione" appiattiva la figura dello scrittore mentre proprio su di lui incombeva la responsabilità di riproporre il passato, traducendolo non solo dal latino, ma dal manoscritto al libro stampato; lo scrittore diventava quindi un traduttore di lingua e di cultura, un investigatore, uno scrutatore, un compilatore o, ma con senso tutto cinquecentesco, un operatore culturale. I grandi scrittori del passato in questo contesto assumono una dimensione ancora più imponente, diventano, se già non lo sono, figure mitiche, perché si accentua la distanza tra loro e gli intellettuali del presente. Petrarca, per fare un esempio, certamente non aveva avuto bisogno di farsi strada a gomitate come lo Scaligero per darsi un'identità, 
perché era davvero nella torre d'avorio dei rari e preziosi ingegni nutriti dal "tutto" contenuto nei codici manoscritti. Nei libri a stampa ci sono invece i "pezzi" sempre più piccoli di un "tutto" sempre più grande. E sono proprio questi frammenti in cui il libro medievale della natura si squaderna che vengono privilegiati nella nuova e tipografica percezione del reale.

\section{University of Toronto}

\section{OPERE CITATE}

Campanella, Tommaso. La città del Sole. Ed. Adriano Seroni. Milano: Feltrinelli, 1983.

Candela, Giuseppe. Manierismo e condizioni della scrittura in Anlon Francesco Doni. New York: Peter Lang, 1993.

Cardano, Gerolamo. Della mia vila. Ed. Alfonso Ingegno. Milano: Serra e Riva 1982.

Cherchi, Paolo. Enciclopedismo e politica della riscrittura. Pisa: Pacini, 1980.

Cocchiara, Giuseppe. Il mondo alla rovescia. Presentazione di Piero Camporesi. Torino: Boringhieri, 1981.

de Kerckhove, Derrick. Brainframes. Utrecht: BSO/Origin, 1991. Verlag, 1988. and Charles J. Lumsden, eds. The Alphabet and the Brain. Heidelberg: Springer"Introduction à la recherche neuroculturelle". McLuhan e la metamorfosi dell' uomo. Ed. Derrick de Kerckhove e Amilcare Iannucci. Roma: Bulzoni, 1984. 147-89.

Garzoni, Tomaso. Opere. Ed. Paolo Cherchi. Ravenna: Longo, 1993. . Opere. Ed. Paolo Cherchi. Napoli: Fulvio Rossi, 1972.

Le vile delle donne illustri della Scrillura Sacra. Ed. Beatrice Collina. Ravenna: Longo, 1994.

L'huomo astratlo. Venezia: Ciotti, 1604. (Ristampa anastatica: Ravenna: Essegi, 1989). Il serraglio de gli stupori del mondo. Venezia: Ambrosio e Bartolomeo Dei, 1613.

McLuhan, Marshall. The Gutenberg Galaxy: The Making of Typographic Man. Toronto: U of Toronto $\mathrm{P}, 1962$.

1988.

Quondam, Amedeo. "La letteratura in tipografia". Letteratura italiana. Ed. Alberto Asor Rosa. Vol. 2. Produzione e consumo. Torino: Einaudi, 1983. 555-686.

Waters, William George. Jerome Cardan: A Biographical Study. London: Lawrence \& Bullen, 1898. 